\title{
Correction to: Survey on Artificial Intelligence for Vehicles
}

\author{
Jun $\mathrm{Li}^{1} \cdot$ Hong Cheng ${ }^{2} \cdot$ Hongliang Guo ${ }^{2}$ Shaobo Qiu ${ }^{1}$ \\ Published online: 26 September 2018 \\ (c) The Author(s) 2018

\section{Correction to: \\ Automotive Innovation (2018) 1:2-14} \\ https://doi.org/10.1007/s42154-018-0009-9
}

The original version of the abstract and keywords of this article has been revised to incorporate recent updates. The revised abstract and keywords are given below.

\begin{abstract}
This paper surveys the literature of Artificial Intelligence for Vehicles (AIV) and gives out the current status and next generation of AIV. The two branches of artificial intelligence, namely Practical Artificial Intelligence (PAI) and Advanced Artificial Intelligence (AAI) are rigorously reviewed. Two of the currently prevailing AIV use cases such as autonomous vehicles and connected vehicles are reviewed, and the key technologies in AIV such as world model, planner and decision maker, computing platform and canonical AV use cases are presented in the paper. The paper also serves as a guidance for the future development of artificial intelligence for vehicles.
\end{abstract}

Keywords Artificial intelligence - Deep neural networks · Connected vehicles $\cdot$ Intelligent vehicles

Open Access This article is distributed under the terms of the Creative Commons Attribution 4.0 International License (http://creativecomm ons.org/licenses/by/4.0/), which permits unrestricted use, distribution, and reproduction in any medium, provided you give appropriate credit to the original author(s) and the source, provide a link to the Creative Commons license, and indicate if changes were made.

The original article can be found online at https://doi.org/10.1007/s42 154-018-0009-9.

Hongliang Guo

guohl1983@uestc.edu.cn

1 China FAW Corporation Limited R\&D Center, Changchun 130000, China

2 Center for Robotics, University of Electronic Science and Technology of China, Chengdu 610000, China 